\title{
Anniversaries, peroxisomes and reactive oxygen species
}

\author{
Michael Schrader
}

Published online: 21 February 2009

(C) Springer-Verlag 2009

In 2008, the annual symposium of the Society for Histochemistry was held in Interlaken, Switzerland (1-4 October). It was not only the 50th symposium of the Society for Histochemistry, but also the 50th anniversary of Histochemistry and Cell Biology. Initially, I was invited by Jürgen Roth and the organizers to give a seminar on peroxisomes in a session on cellular defense mechanisms. When I became aware that the session was supposed to be chaired by H. Dariush Fahimi (the former supervisor of my $\mathrm{PhD}$ thesis) who was about to celebrate his 75th anniversary in 2008 , I communicated this coincidental observation to the organizers. We spontaneously decided to celebrate $\mathrm{H}$. Dariush Fahimi's anniversary as well (without telling him!), and the session was renamed "Peroxisomes, ROS and HDF 75". Furthermore, colleagues from the "early days" of peroxisome research were invited to make a contribution to the session (Fig. 1). Although scheduled for 8:00 am on a cloudy and rainy Friday morning, the topic attracted many participants and congratulators and led to vivid discussions. Subsequently, the speakers of the session were encouraged by the editors of this journal to summarize their talks in the form of a brief report or mini-review and to give a short overview and/or perspective of their field of interest.

As an introduction to those reports, H. Dariush Fahimi (Heidelberg, Germany; Fig. 1) delineates how he contributed to the introduction of the DAB-method for light and electron microscopic visualization of peroxisomes. Coincidentally, the 50th anniversary symposium also commemo-

M. Schrader ( $\square)$

Centre for Cell Biology and Department of Biology,

University of Aveiro, 3810-193 Aveiro, Portugal

e-mail:mschrader@ua.pt rated the 40th year of the introduction of this elegant method, which led to the discovery of peroxisomes as a ubiquitous eukaryotic organelle. It was revealed that the peroxidatic activity of catalase, an abundant peroxisomal marker enzyme, is responsible for that staining. The discovery of the co-localization of several $\mathrm{H}_{2} \mathrm{O}_{2}$-producing oxidases, together with catalase in peroxisomes, was the first indication suggesting the participation of peroxisomes in the metabolism of oxygen metabolites. It also led Christian de Duve to propose the term "peroxisome" for the designation of that organelle.

In our brief report (Delille et al. this issue), we address the classical and novel views on the formation of peroxisomes, which is again hotly debated. We contributed to the identification of the first components involved in the growth and division of peroxisomes. Interestingly, some of these components (e.g., dynamin-like protein 1, hFis1, Mff) are shared by the division machinery of both peroxisomes and mitochondria. Recently, it became clear that this is a common strategy used by mammals, fungi and plants. We propose a closer interrelationship between peroxisomes and mitochondria, which might have an impact on functionality and disease conditions. Furthermore, peroxisomes and mitochondria have been suggested to contribute to pathological conditions associated with oxidative stress and have as well been linked to aging.

The central role of peroxisomes in the generation and scavenging of $\mathrm{H}_{2} \mathrm{O}_{2}$ has been well known ever since their discovery almost five decades ago. Recent findings on the biochemical properties of catalase and the abundant peroxisomal oxidases (e.g., D-amino acid oxidase, urate oxidase, xanthine oxidase) are highlighted by Alfred Völkl et al. (Heidelberg, Germany; Fig. 1; Angermüller et al. this issue). The cerium technique and immunoelectron microscopy have been successfully used to localize these enzymes in peroxi- 

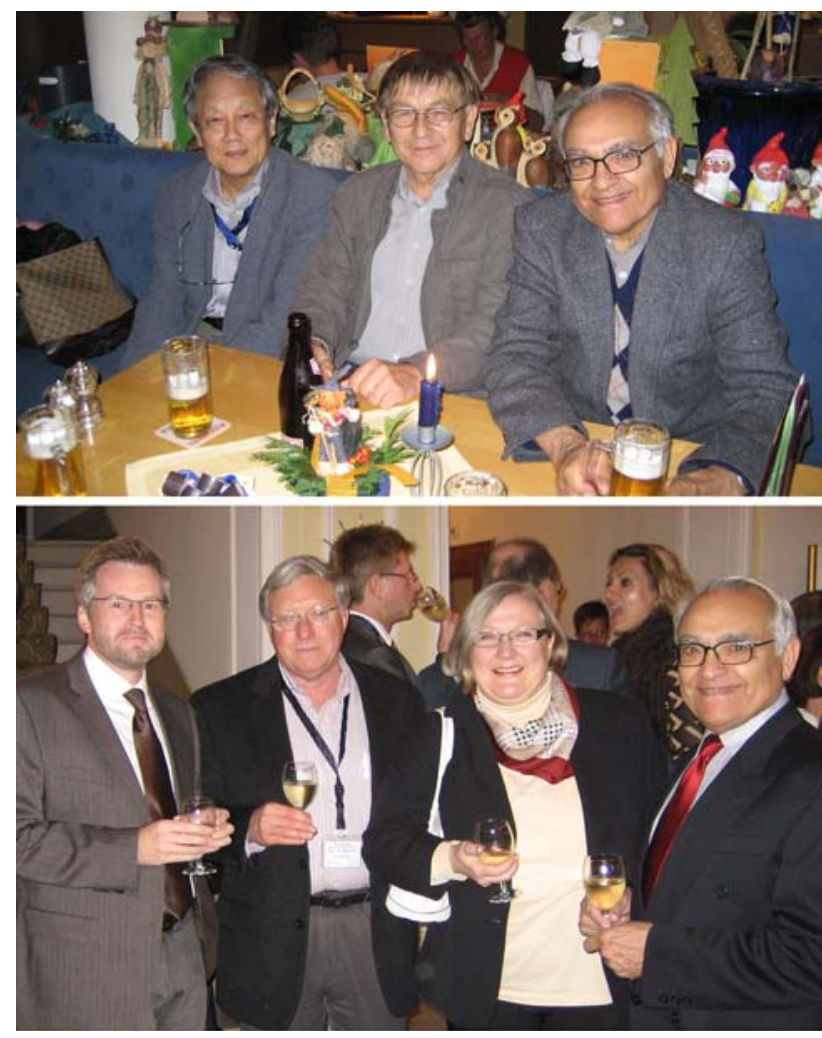

Fig. 1 Speakers and congratulators of the session on "Peroxisomes, ROS and HDF 75" at the 50th symposium of the Society for Histochemistry, Interlaken, Switzerland, October 1-4. From left to right: Sadaki Yokota (Nagasaki, Japan), Alfred Völkl (Heidelberg, Germany) and H. Dariush Fahimi (Heidelberg, Germany) in a local pub (top). Michael Schrader (Aveiro, Portugal), John M. Robinson (Columbus, USA), Eveline Baumgart-Vogt (Giessen, Germany), and H. Dariush Fahimi (Heidelberg, Germany) at the symposium reception (bottom)

somes and their crystalline inclusions. Coincidentally, Alfred Völkl was celebrating his 68th anniversary during the symposium in Interlaken (this was done more privately with an excellent bottle of Port wine directly from Oporto).

John M. Robinson (Columbus, USA; Fig. 1) reports on phagocytic leukocytes and ROS production. When stimu- lated, phagocytic leukocytes display a respiratory burst in which they consume oxygen and produce superoxide anions via the phagocyte NADPH-oxidase system. John M. Robinson focuses on the important role of microscopy in complementing biochemical studies to better understand the biology of the respiratory burst. By the way, John Robinson, similar to H. D. Fahimi, also spent many years in the laboratory of Morris J. Karnovsky at Harvard Medical School where many new methods for cytochemical detection of ROS were developed.

Srikanth Karnati (a travel awardee of the Society for Histochemistry from the group of Eveline Baumgart-Vogt, Giessen, Germany; Fig. 1) presents his results on the abundance and distribution of peroxisomal marker proteins in distinct cell-types and developmental stages of the murine and human lung (original article by Karnati and BaumgartVogt, this issue). It is assumed by the authors that peroxisomes might play an important role in pulmonary biology.

Finally, Sadaki Yokota (Nagasaki, Japan; Fig. 1) discusses the mechanisms for the degradation of excess peroxisomes in mammalian hepatocytes (Yokota and Fahimi, this issue). These mechanisms include autophagy, the possible role of a peroxisome-specific isoform of Lon protease, and the selective, membrane-disrupting effect of 15-lipoxygenase, which is followed by diffusion of matrix proteins and proteolysis in the cytoplasm.

Once again, I would like to thank the editors, speakers and authors for their support, valuable time, efforts and contributions. I hope that the readers of Histochemistry and Cell Biology will enjoy this collection of brief reports on peroxisomes and ROS. Should you get interested in the biology of peroxisomes and decide to read more about this fascinating, but still mysterious, organelle, I can strongly recommend a collection of reviews published in a special issue of Biochim Biophys Acta (vol 1763, 2006) as well as a recent open access review in this journal (Schrader and Fahimi (2008) Histochem Cell Biol 129(4):421-40). 